\title{
Chronic immobilization stress occludes in vivo cortical activation in an animal model of panic induced by carbon dioxide inhalation
}

\author{
Mohammed Mostafizur Rahman ${ }^{1}$, Christian M. Kerskens ${ }^{2}$, Sumantra Chattarji ${ }^{1}$ and \\ Shane M. O'Mara ${ }^{2 *}$
}

${ }^{1}$ National Center for Biological Sciences, Tata Institute of Fundamental Research, Bangalore, India

2 Trinity College Institute of Neuroscience, Trinity College Dublin, Dublin, Ireland

\section{Edited by:}

Regina M. Sullivan, Nathan Kline Institute and New York University School of Medicine, USA

\section{Reviewed by:}

Francesca R. D'Amato, National

Research Council, Italy

Daniel W. Wesson, Case Western

Reserve University, USA

*Correspondence:

Shane M. O'Mara, Trinity College Institute of Neuroscience, Trinity College Dublin, Lloyd Building, Dublin 2, Ireland

e-mail:shane.omara@gmail.com
Breathing high concentrations of carbon dioxide $\left(\mathrm{CO}_{2}\right)$ can trigger panic and anxiety in humans. $\mathrm{CO}_{2}$ inhalation has been hypothesized to activate neural systems similar to those underlying fear learning, especially those involving the amygdala. Amygdala activity is also upregulated by stress. Recently, however, a separate pathway has been proposed for interoceptive panic and anxiety signals, as patients exhibited $\mathrm{CO}_{2}$-inhalation induced panic responses despite bilateral lesions of the amygdala. This paradoxical observation has raised the possibility that cortical circuits may underlie these responses. We sought to examine these divergent models by comparing in vivo brain activation in unstressed and chronically-stressed rats breathing $\mathrm{CO}_{2}$. Regional cerebral blood flow measurements using functional Magnetic Resonance Imaging (fMRI) in lightly-anaesthetized rats showed especially strong activation of the somatosensory cortex by $\mathrm{CO}_{2}$ inhalation in the unstressed group. Strikingly, prior exposure to chronic stress occluded this effect on cortical activity. This lends support to recent clinical observations and highlights the importance of looking beyond the traditional focus on limbic structures, such as the hippocampus and amygdala, to investigate a role for cortical areas in panic and anxiety in humans.

Keywords: carbon dioxide, hypercapnia, Magnetic Resonance Imaging, panic, somatosensory cortex, stress

\section{INTRODUCTION}

DSM-V defines panic attacks as "A discrete period of intense fear or discomfort, in which four (or more) of the enlisted symptoms (such as sweating, chest pain, paresthesias, trembling, nausea etc.), developed abruptly and reached a peak within 10 min" (American Psychiatric Association et al., 2013). Inhaling carbon dioxide $\left(\mathrm{CO}_{2}\right)$ induces an emotional response similar to fear and panic attacks (Papp et al., 1997; Griez and Schruers, 1998). In healthy individuals inhaling high amounts of $\mathrm{CO}_{2}$ (20\%) induces panic attacks (Forsyth et al., 2000). Panic disorder patients show panic response to even small doses of $\mathrm{CO}_{2}$ inhalation (Gorman et al., 1994). Patients with anxiety disorders or a family history of anxiety disorder are also more susceptible to show panic response to $\mathrm{CO}_{2}$ inhalation (Coryell, 1997). Inhaling hypercapnic gases, such as various concentrations of $\mathrm{CO}_{2}$, has been hypothesized to trigger a biological alarm system that has evolved to serve as a suffocation monitor (see Preter and Klein, 2008 for a review) (Klein, 1993; Preter and Klein, 2008). Earlier studies have tried to infer the neural circuit involved in this panic response. The hippocampus, amygdala and cortex (Sakai et al., 2005; Ziemann et al., 2009) have all been hypothesized to play a vital role in $\mathrm{CO}_{2}$-induced panic response. Some studies suggest that the panic response is similar to that caused by activation of the fear pathway (Gorman et al., 2000). Moreover, the amygdala can act as a chemosensor for $\mathrm{pH}$ changes, thereby regulating it's response to $\mathrm{CO}_{2}$ inhalation (Ziemann et al., 2009). Together these findings suggest a potential role for the amygdala in $\mathrm{CO}_{2}$-induced panic response. However, a recent study has reported $\mathrm{CO}_{2}$-induced panic response in human patients with amygdala lesions arising from Urbach-Wiethe disease (Feinstein et al., 2013). This hints at a potential parallel pathway for triggering intrinsic fear due to $\mathrm{CO}_{2}$-inhalation, possibly interoceptive in nature (see Critchley, 2005 for a review) (Critchley, 2005).

Stress-related psychiatric disorders have been shown to cause anxiety and enhanced fear responses in humans (Morgan III et al., 1995; Groome and Soureti, 2004). Since it has been hypothesized that panic response is similar to that caused by activation of the fear pathway, stress might show an influence on the panic response caused by $\mathrm{CO}_{2}$ inhalation. However, little is known about the interaction between stress and $\mathrm{CO}_{2}$-inhalation. Strengthening of the structural and physiological basis of synaptic connectivity in the amygdala has been implicated in chronic stress induced increase in anxiety and fear (Mitra et al., 2005; Suvrathan et al., 2014). However the fact that panic response due to $\mathrm{CO}_{2}$ inhalation involves the amygdala (Ziemann et al., 2009), but can also occur without amygdalar involvement (Feinstein et al., 2013), making it an intriguing case for further investigation. Therefore, we performed functional Magnetic Resonance Imaging (MRI) during the inhalation of $\mathrm{CO}_{2}$ in lightly-anaesthetized rats that 
were either unstressed or previously exposed to 10 days of chronic stress.

\section{MATERIALS AND METHODS EXPERIMENTAL ANIMALS}

Twelve adult male Wistar rats (Trinity College Dublin BioResources Unit) weighing $270-300 \mathrm{~g}$ were used in the study. The animals were housed in groups of two. Animals were kept on a 12/12-h light/dark cycle and had access to water and a standard diet ad-libitum. All experiments were conducted in accordance with protocols approved by the Animal Ethics Committee of Trinity College, Dublin. These procedures were licensed by the Irish Department of Health and Children.

\section{STRESS PROTOCOL}

The behavioral stress protocol has been described in several studies (Mitra et al., 2005). Briefly, rats were randomly assigned to experimental groups-stressed or unstressed. Rats in the stressed group were subjected to a chronic immobilization stress (CIS) paradigm. The CIS paradigm consisted of complete immobilization for $2 \mathrm{~h}$ per day (before noon) in rodent immobilization bags without access to either food or water, for 10 consecutive days. On the 11th day the rats were subjected to the $\mathrm{CO}_{2}$ inhalation test (Figure 1B).

\section{ANIMAL PREPARATION}

All animals were anaesthetized with 5\% isoflurane (Isoflo, Abbott, Queenboro, England) in oxygen $(1 \mathrm{~L} / \mathrm{min}$ ) and maintained with $1.5-2 \%$ isoflurane. The level of anesthesia was regularly monitored throughout the procedure using the pedal withdrawal reflex to toe pinch. Tail vein blood was collected just after anesthetizing the rats as well as at the end of the experiment, to obtain blood gas samples. These samples were used to measure the concentration of carbon dioxide in blood before and after the experiment. We found no change in carbon dioxide concentration in the blood before and after the experiment. After the collection of samples the animals were placed prone in a Plexiglas cradle with a three point-fixation system (tooth-bar and ear pieces). Temperature was maintained constant at $37^{\circ} \mathrm{C}$ using a warming surface controlled by a water pump-driven temperature regulator. The respiration signal was monitored using custom hardware and software (SA Instruments Inc., Stony Brook, NY, USA). The animals were then placed in a $7 \mathrm{~T}, 30 \mathrm{~cm}$ bore animal MRI system (Biospec 70/30, Bruker Biospin, Ettlingen, Germany) scanner with a circular polarized $1 \mathrm{H}$ rat brain $\mathrm{RF}$ coil (Bruker, BioSpin). A $7 \mathrm{~cm}$ diameter volume coil was used for transmission of the CASL and FLASH excitation pulses. Signal detection was performed using a surface coil.

\section{$\mathrm{CO}_{2}$-INHALATION PROTOCOL}

$\mathrm{CO}_{2}$ was mixed with oxygen (hyperoxic) and the percentage composition was maintained using partial pressure of the two gases. This mixture $(1 \mathrm{~L} / \mathrm{min})$ replaced the oxygen supply. The amount of $\mathrm{CO}_{2}$ was maintained at the levels of $0,7,10,15$, and $20 \%$ in increasing order, with each concentration maintained for $5 \mathrm{~min}$ before changing the concentration. Finally, the amount of $\mathrm{CO}_{2}$ was decreased to $0 \%$ (Figure 1B).

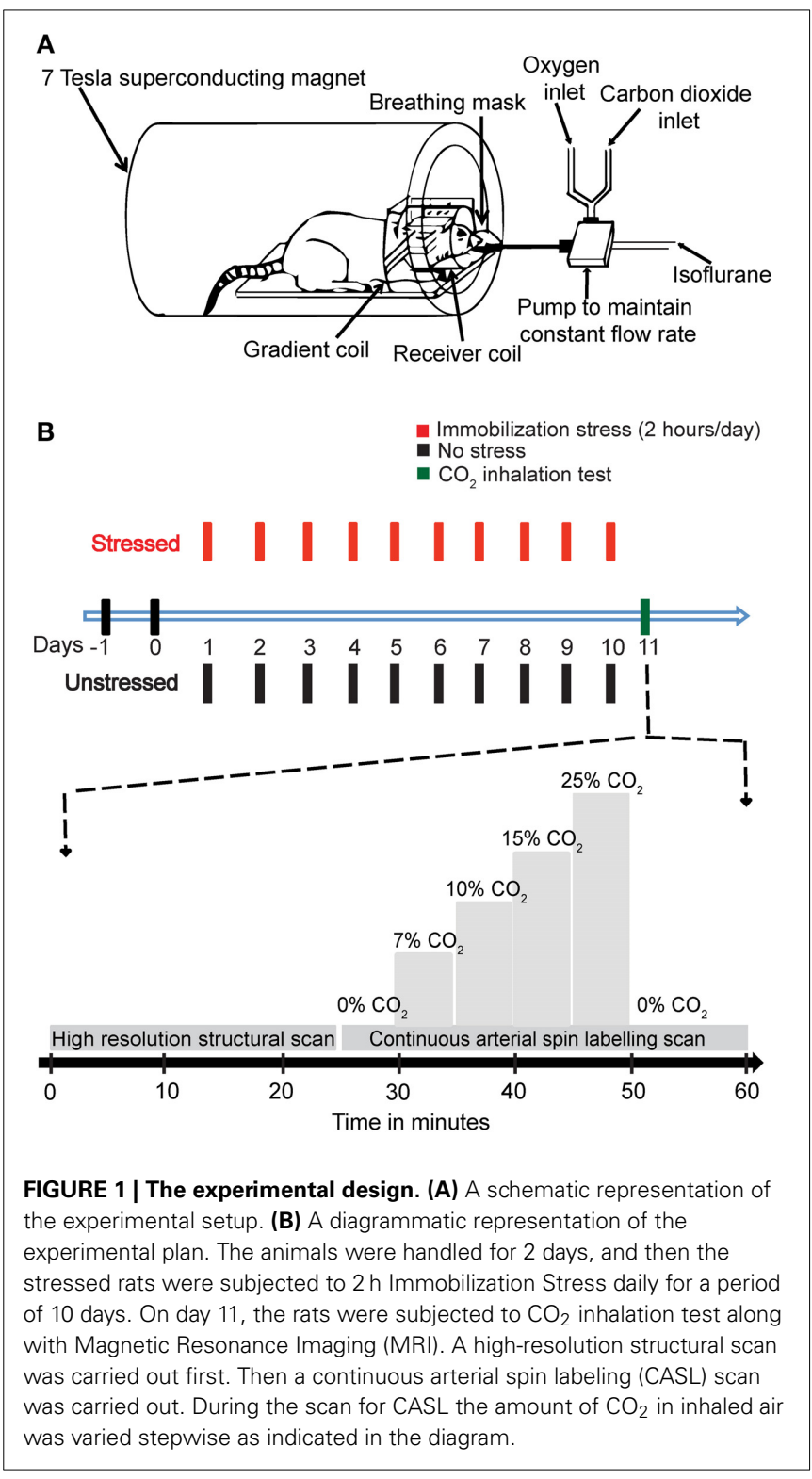

\section{HIGH RESOLUTION ANATOMICAL SCANS}

A rapid acquisition with relaxation enhancement (RARE) (Hennig et al., 1986) high resolution anatomical scan (slice thickness $=0.5 \mathrm{~mm}, T E=36.7 \mathrm{~ms}, T R=5.4595 \mathrm{~s}, \mathrm{FOV}=4 \times 4 \mathrm{~cm}$, image matrix $=256 \times 256$, RARE factor $=4$ ) was performed and compared to a rat brain atlas in order to locate the slice with optimal somatosensory cortex, motor cortex, hippocampus and amygdala coverage (Figure 2A; Paxinos and Watson, 2005). This imaging slice location (thickness $=2.0 \mathrm{~mm}$ ) was then used for the subsequent ASL sequence.

\section{ARTERIAL SPIN LABELING (ASL) MRI SEQUENCE}

The ASL sequence consisted of a 5-s preparation interval containing the inversion pulse, followed by a snap shot fast low-angle shot (FLASH) image acquisition [echo time $[\mathrm{TE}]=1.67 \mathrm{~ms}$, repetition time $[\mathrm{TR}]=5.0 \mathrm{~ms}$, flip angle $[\mathrm{FA}]=20^{\circ}$, bandwidth 


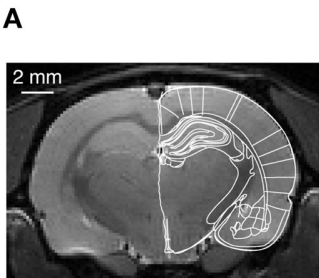

High resolution structural Image

B

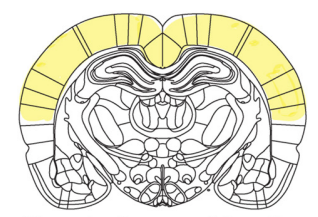

Stereotactic map of the slice

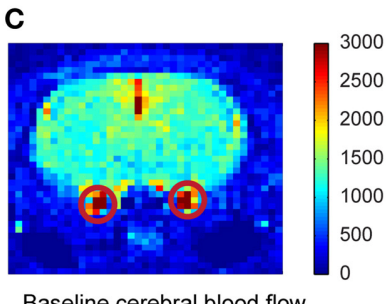

Baseline cerebral blood flow
D

(i)

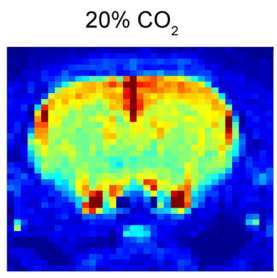

(ii)

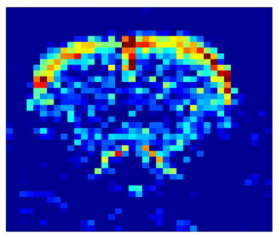

(iii)

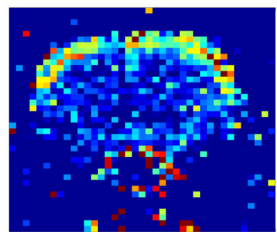

(iv)

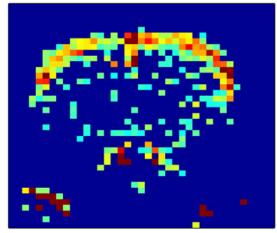

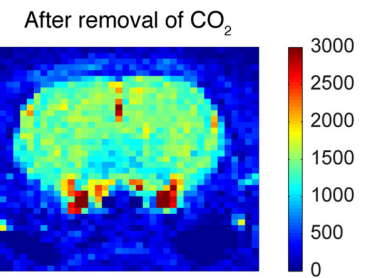
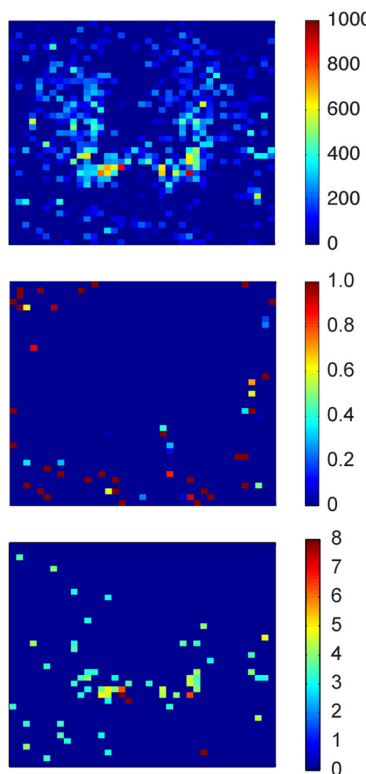

FIGURE 2 | Cerebral blood flow (CBF) pattern caused by inhalation of $\mathbf{C O}_{\mathbf{2}}$. (A) High resolution structural image of the slice acquired prior to $\mathrm{CO}_{2}$ inhalation. (B) Coronal plate from "The rat brain in stereotaxic coordinates" (Paxinos and Watson, 2005). Plate coordinates: $-3.0 \mathrm{~mm}$ from bregma. All the imaging was done in the corresponding coronal slice plane. The region marked in yellow color represents mainly the motor cortex and somatosensory cortex. (C) Baseline CBF before the inhalation of $\mathrm{CO}_{2}$ of one of the animals. The colorbar represents the intensity of signal in a.u., which is directly proportional to regional cerebral blood volume (rCBV). The red circles show the carotid artery. (D) Transient CBF pattern caused by the inhalation of $\mathrm{CO}_{2}$. (i) The CBF during $20 \% \mathrm{CO}_{2}$ inhalation and after removal of $\mathrm{CO}_{2}$. The colorbar represents values in a.u., which is directly proportional to rCBV. (ii) Subtracted images (activation-baseline) showing increased CBF only. (iii) Increase in CBF normalized to the baseline CBF. (iv) Z-score pixel wise (only pixels having z-score above 3 are represented here).
$[\mathrm{BW}]=100 \mathrm{kHz}$, number of repetitions $[\mathrm{NR}]=16$, slice thickness $=2.0 \mathrm{~mm}$, number of slices $=1$, field of view $[\mathrm{FOV}]=3.0 \times$ $3.0 \mathrm{~cm}$, matrix $=64 \times 64$, acquisition time $=5.082 \mathrm{~s}$ per repetition (Kerskens et al., 1996)]. Flow-induced fast adiabatic passage of inflowing inverted arterial spins was performed using a rectangular pulse. Inverted arterial spins then travel to the imaging plane (Dixon et al., 1986; Kelly et al., 2009). The inversion pulse radio frequency power $(-22 \mathrm{~dB})$ and offset frequency $(-12 \mathrm{kHz})$ were determined to give optimal perfusion contrast by achieving inversion $2 \mathrm{~cm}$ proximal to the imaging plane. Control images with the offset frequency reversed $(12 \mathrm{kHz})$, in which inflowing spins were left undisturbed, were also acquired, in an interleaved fashion. Eight repetitions of each image type were acquired for signal averaging using ParaVision 4.0 software (Bruker Biospin) for data reconstruction and analysis. Each individual 5-min segment of increasing $\mathrm{CO}_{2}$ concentration consisted of:

(i) Two minutes were given for the $\mathrm{CO}_{2}$ to reach the animal and get inhaled after increasing the $\mathrm{CO}_{2}$ concentration. (ii) Following this 2-min segment, $1.5 \mathrm{~min}$ were devoted to doing the scans.

(iii) The final $1.5 \mathrm{~min}$ was provided for the animal to rest before the next increase in $\mathrm{CO}_{2}$ concentration was initiated.

Ten minutes were provided for the animals to recover back to baseline before scanning for the $0 \% \mathrm{CO}_{2}$ concentration was done. Then the next increase in concentration was started.

\section{QUALITATIVE ASL DATA ANALYSIS}

Arterial spin images were exported to TIFF format from the $2 \mathrm{dseq}$ format (Bruker format, Bruker Biospin, Ettlingen, Germany) using ImageJ (US. National Institutes of Health, Bethesda, Maryland, USA) (Schneider et al., 2012). The exported images were subsequently processed and analyzed using custom written codes in MATLAB (MathWorks, Inc., USA). There were 4 scan sessions for each $\mathrm{CO}_{2}$ concentration. For each scan session 16 images were captured, 8 images with spin labeling and 8 corresponding control images. The two stacks were averaged and the average labeled image was subtracted from the average control 
image to get a result image for the corresponding scan (Griffin et al., 2010). The resulting images formed for each concentration were averaged to get the activation perfusion image at that concentration. The baseline perfusion image $(0 \%$ concentration of $\mathrm{CO}_{2}$ ) was subtracted from the perfusion image at a certain concentration to obtain the change in perfusion induced by the $\mathrm{CO}_{2}$ inhalation. The change in perfusion image was normalized to the baseline image, in a pixel-by-pixel manner, to obtain the activation map due to $\mathrm{CO}_{2}$ inhalation (Figure 2D). The baseline perfusion and the perfusion image at a particular concentration were also used to calculate the z-score in a pixel-by-pixel manner. These were then threshold at $\mathrm{z}$-score of 3 (Figure 2Div).

\section{QUANTIFICATION OF ASL DATA}

The activation maps obtained by the method mentioned above were quantified to obtain the activation area and activation intensity. Intensity threshold was used in the baseline image to demarcate the brain slice. The number of pixels in the demarcated area was counted to calculate the total slice area. All the pixels in the demarcated area were used to calculate the root mean square noise (RMS noise) for each image, using the formula:

$$
\text { RMSnoise }=\sqrt{\frac{\sum_{i=1}^{n}\left(X_{i}-\frac{\sum_{i=1}^{n} X_{i}}{n}\right)^{2}}{n}}
$$

where $n=$ total number of pixels, $X_{i}$ is the intensity of each pixel.

Activation threshold was set at $2 \times$ RMSnoise. Any pixel having intensity more than the threshold was considered an activated pixel. The normalized activation intensity was calculated by adding up the pixel intensities of all the activated pixels (Figure 3D). The total activation area was calculated by counting the number of activated pixels (Figure 3C). The activation intensity per pixel was obtained by dividing the normalized activation intensity with the activation area. The normalized activation area was calculated by dividing total activation area with the total slice area (Figure 3E).

\section{STATISTICAL ANALYSIS}

All statistical analysis was carried out using GraphPad prism (GraphPad software Inc., La Jolla, CA, USA). All results are presented as mean \pm SEM unless otherwise stated. Statistical analysis was performed using repeated measures Two-Way ANOVA, unless otherwise stated. The post-hoc test performed was HolmSidak's test, unless otherwise mentioned. The criterion for statistical significance was $p<0.05$.

\section{RESULTS}

In the current study, we used two groups of animals-control unstressed rats and rats that were subjected to 10 days of chronic immobilization stress ( $2 \mathrm{~h} /$ day) (Figure 1B top; Materials and Methods). The stressed animals showed a lack in bodyweight gain as compared to the unstressed animals (Supplementary Figure S1). This is in agreement with earlier studies confirming the efficacy of the chronic immobilization stress protocol (Vyas et al., 2002; Lakshminarasimhan and Chattarji, 2012). On the 11 th day (i.e., $24 \mathrm{~h}$ after the end of the 10-day chronic stress paradigm), both groups of rats underwent fMRI (Figure 1A;
Materials and Methods) during inhalation of varying levels of $\mathrm{CO}_{2}$ (Figure 1A bottom; Materials and Methods). To this end we focused on brain regions implicated in previous studies (Sakai et al., 2005; Ziemann et al., 2009; Gozzi et al., 2010). Therefore, we selected a brain slice that contained the amygdala, hippocampus and somatosensory cortex (Figure 2A). We first obtained a baseline perfusion map for the slice followed by a map during $\mathrm{CO}_{2}$ inhalation by the same rat (Figure 2C).

\section{INHALATION OF $\mathrm{CO}_{2}$ TRIGGERS CORTICAL ACTIVATION}

We observed an increase (15\% relative to baseline) in perfusion (i.e., cerebral blood flow) across the entire brain sliceduring $\mathrm{CO}_{2}$ inhalation in unstressed rats (Figure 2D). Moreover, the increase in perfusion in the somatosensory cortex was greater $(50 \%$ relative to baseline) than the whole-slice value. Thus, $\mathrm{CO}_{2}$-inhalation led to increased cortical activity. This activity pattern was transient and disappeared on removal of $\mathrm{CO}_{2}$ from the breathing gas mixture. Similar increases in perfusion have been reported due to increase in activity of neurons following forepaw stimulation (Griffin et al., 2010) suggesting that the increases we observe are within normal physiological ranges. In addition to this thresholdbased analysis (Figures 2, 3) we also carried out an analysis based on z-scores. This analysis further confirms cortical activation $\mathrm{CO}_{2}$-inhalation (Supplementary Figure S2).

\section{STRESSED RATS SHOW LOWER CORTICAL ACTIVATION ARISING FROM $\mathrm{CO}_{2}$ INHALATION COMPARED TO UNSTRESSED RATS}

Both stressed and unstressed rats show increased cortical activation due to $\mathrm{CO}_{2}$-inhalation. However, the activation patterns are markedly different between the groups. The overall activation is less for the stressed group (Figure 3B, Supplementary Figure S2) and this pattern was evident across all $\mathrm{CO}_{2}$ concentrations tested $(7,10,15$, and $20 \%)$.

\section{QUANTIFICATION OF ACTIVATION CAUSED BY $\mathrm{CO}_{2}$-INHALATION}

We carried out a more detailed quantification of the changes in activation pattern based on four parameters - normalized activation intensity, activation intensity per pixel, activation area and normalized activation area. An activation threshold was used to determine the activated pixels, which in turn were used to calculate these parameters (Materials and Methods). We used the normalized activation images for all the quantification, as these images give a more direct measure of cerebral blood flow (CBF) as compared to the z-score images (Griffin et al., 2010).

Normalized activation intensity is the sum of the normalized (to the baseline perfusion) intensities of all activated pixels. A high value for normalized activation intensity means high levels of cerebral blood flow. We see significantly higher levels of cerebral perfusion in unstressed rats compared to the stressed rats (Two-way repeated measures ANOVA, factor: stress, $p<$ 0.05) (Figure 3D). The significant difference holds for all the $\mathrm{CO}_{2}$ concentrations used (post-hoc Holm-Sidak's multiple comparison test). Further, while unstressed rats show a significant decrease $(p<0.0001)$ in perfusion on removal of $\mathrm{CO}_{2}$ (post-hoc Sidak's multiple comparison test), stressed rats do not exhibit this decrease on removal of $\mathrm{CO}_{2}$. There were no dose-dependent differences in perfusion for either unstressed or stressed rats. 


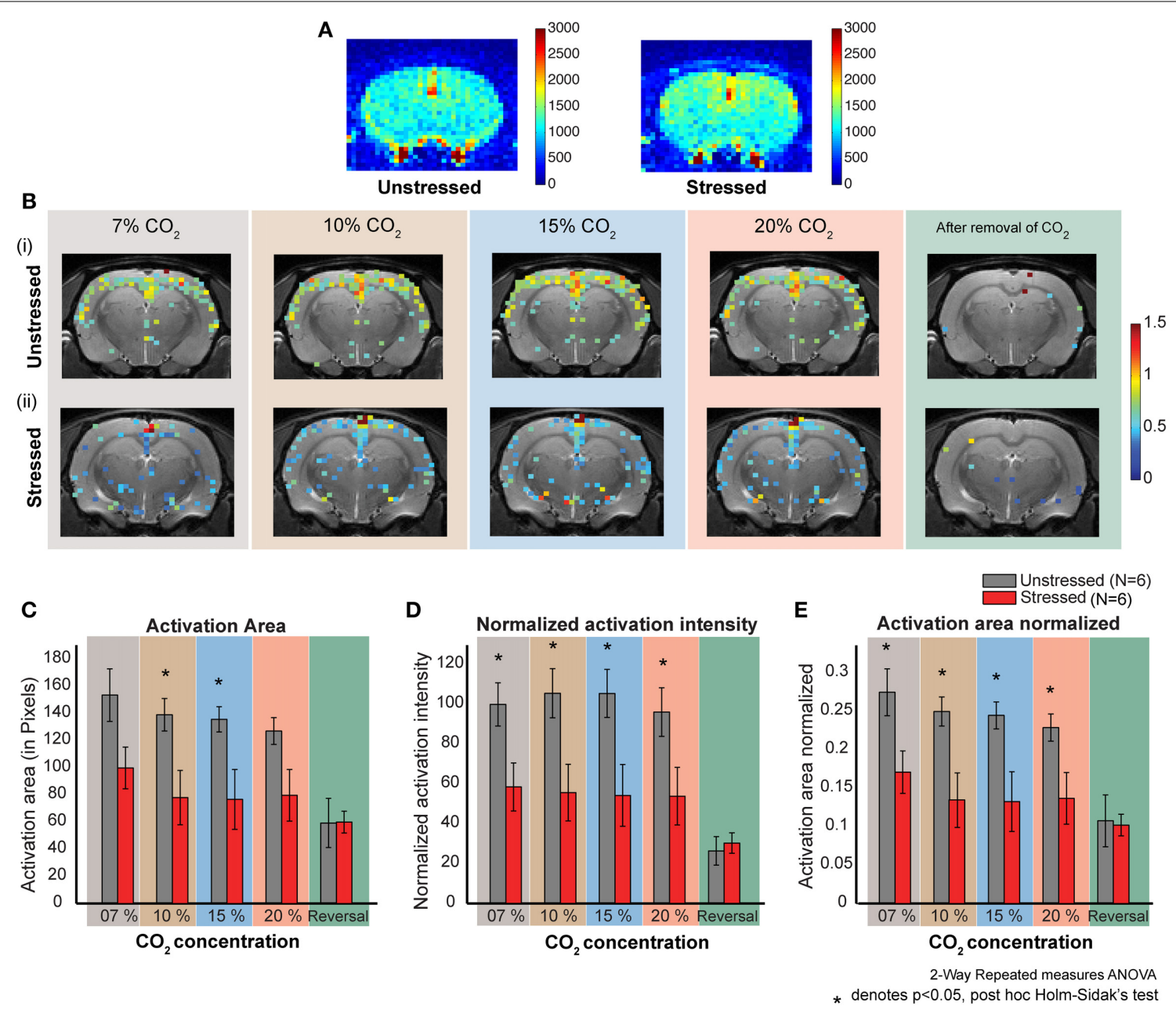

FIGURE 3 | $\mathrm{CO}_{2}$ induced dose dependent $\mathrm{CBF}$ in stressed and unstressed animals. (A) Baseline CBF before the inhalation of $\mathrm{CO}_{2}$. The left image is from an unstressed animal and the right is from a stressed animal. The colorbar represents the intensity of signal in a.u., which is directly proportional to regional cerebral blood volume (rCBV). (B) Dose dependent response to $\mathrm{CO}_{2}$ inhalation. Each image represents the activation (increase in CBF normalized to the baseline) due to the inhalation of indicated percentage of $\mathrm{CO}_{2}$. (i) Represents images from an unstressed animal. (ii) Represents images from a stressed animal. (C-E) Quantification of response to $\mathrm{CO}_{2}$ inhalation in unstressed $(N=6)$ and stressed $(N=6)$ animals. (D)

Normalized activation intensity (which is directly proportional to increase in cerebral blood flow normalized to baseline cerebral blood flow) in response to $\mathrm{CO}_{2}$ inhalation. There is a significant effect of stress $(p=0.0261)$ and $\mathrm{CO} 2$ inhalation ( $p<0.0001$ ). The response of unstressed and stressed rats is significantly different for all the concentrations $\mathrm{CO}_{2}$. (C) Activation area in response to $\mathrm{CO}_{2}$ inhalation. There is a significant effect of stress $(p=0.0404)$ and $\mathrm{CO}_{2}$ inhalation $(p<0.0001)$. The response of unstressed and stressed rats is significantly different for $10 \%$ and $15 \%$ concentrations of $\mathrm{CO}_{2}$. (E) Activation area normalized to the total slice area in response to $\mathrm{CO}_{2}$ inhalation. There is a significant effect of stress $(p=0.0264)$ and $\mathrm{CO}_{2}$ inhalation ( $p<0.0001$ ). The response of unstressed and stressed rats is significantly different for all the concentrations of $\mathrm{CO}_{2}$.
Activation intensity per pixel is the activation intensity normalized to the number of pixels in the slice. This is a measure of perfusion density, i.e., perfusion per pixel. We did not see any significant difference in perfusion density between unstressed and stressed rats (data not shown). However, perfusion density does decrease significantly after the removal of $\mathrm{CO}_{2}$ only in the unstressed rats $(p<0.05)$.

Activation area is the total brain area activated due to $\mathrm{CO}_{2}$ inhalation. We see a significantly higher activation area in unstressed, compared to stressed, rats (Two-Way repeated measures ANOVA, factor: stress $p=0.0404$ ) for $10 \%$ and $15 \%$ $\mathrm{CO}_{2}$ (Figure 3C). There is no significant difference for the other concentrations (post-hoc Holm-Sidak's multiple comparison test). Further, unstressed rats show a significant decrease in activation area on removal of $\mathrm{CO}_{2}$, whereas the stressed rats do not show a significant decrease after $\mathrm{CO}_{2}$ removal (post-hoc Sidak's multiple comparison test). There was no dose-dependent difference in activation area for either unstressed or stressed rats.

Normalized activation area is the total area activated due to the inhalation of $\mathrm{CO}_{2}$ normalized to the total slice area. We see a significantly higher activation area in unstressed, compared to the stressed, rats (Two-way repeated measures ANOVA, factor: stress $p$-value $=0.0264)$ (Figure $3 \mathrm{E})$. The significant difference holds for all the concentrations applied (post-hoc Holm-Sidak's 
multiple comparison test). The unstressed rats show a significant decrease in normalized activation area on removal of $\mathrm{CO}_{2}$, but the stressed rats do not show any significant decrease (post-hoc Sidak's multiple comparison test). There was no dose-dependent difference in normalized activation area for either unstressed or stressed rats.

Taken together, two patterns of difference emerge between control and stressed rats. First, inhalation of $\mathrm{CO}_{2}$ elicits an increase in all parameters, except perfusion density, in unstressed rats compared to their stressed counterparts. Second, removal of $\mathrm{CO}_{2}$ leads to a reduction in all the parameters in unstressed, but not stressed, rats. This might be due to the fact that the stressed animals do not show much activation due to $\mathrm{CO}_{2}$ in the first place.

\section{DISCUSSION}

\section{CORTICAL ACTIVATION INDUCED BY $\mathrm{CO}_{2}$-INHALATION}

The present study is one of the first attempts to look at the consequences of two very different types of perturbations that trigger strong emotional effects and how they interact at the level of in vivo neural activity in the intact brain, normally, $\mathrm{CO}_{2}$ inhalation and how these effects interact with exposure to chronic stress. The most striking finding is that $\mathrm{CO}_{2}$ inhalation causes a distinct cortical activation. However we were not able to detect any activation in the amygdala.

We observed an increase in CBF (15\% relative to the baseline) across the slice. This maybe due to a systemic response caused by the vasodilatory effect of $\mathrm{CO}_{2}$. Earlier studies have reported similar levels of $\mathrm{CBF}$ change (less than 10\%) due to vasodilatory effects of $\mathrm{CO}_{2}$ inhalation in humans (Gambhir et al., 1997). However, the activation was the most pronounced in the somatosensory cortex (Figures 2B,D). Similar levels of increase in cerebral blood flow has been obtained due to increases in neuronal activity in earlier studies (Griffin et al., 2010). Therefore we observe activation of the somatosensory cortex due to $\mathrm{CO}_{2}$ inhalation. It is interesting to note that the somatosensory cortex has ASIC1 channels (Wemmie et al., 2003) which can act as chemosensor to $\mathrm{CO}_{2}$ and $\mathrm{pH}$ changes (Chung et al., 2010). Moreover, $\mathrm{CO}_{2}$-inhalation is known to trigger the trigeminal pathway (Wang et al., 2010), and human studies have reported activation of somatosensory cortex among other brain regions due to intranasal trigeminal function (Albrecht et al., 2010).

The primary somatosensory cortex has direct projections to the hypothalamus (Killackey and Sherman, 2003), which plays a pivotal role in maintaining body homeostasis (Williams et al., 2001) and control of the autonomic nervous system (Wang et al., 2007). Interestingly, the somatosensory cortex also projects to the nucleus raphé magnus and periaqueductal gray (Bragin et al., 1984; O'Hearn and Molliver, 1984). While the raphe nucleus is involved in both fear responses (Kiser et al., 1980) and respiratory control (Sessle et al., 1981), the periaqueductal gray mediates defensive behavior (Bittencourt et al., 2005; Schenberg et al., 2005) panic anxiety (Jenck et al., 1995; Schenberg et al., 2001) and suffocation (Kumar et al., 1997) among other autonomic responses. It has been hypothesized that the suffocation alarm system is located within the periaqueductal gray matter (Schimitel et al., 2012). Furthermore, key elements of the panic responses (e.g., increased heart rate, hyperventilation, increased perspiration, etc.), are controlled by the autonomic nervous system (Stein and Asmundson, 1994). Therefore, somatosensory cortex is wellpositioned to serve as a possible candidate substrate to support, and perhaps initiate, the interoceptively-driven panic alarm triggered by $\mathrm{CO}_{2}$ inhalation. It also provides a possible candidate for an alternative pathway for fear and anxiety processing driven by interoceptive stimuli. In this connection, it is interesting to note that using a rat model of near-death experiences caused by cardiac arrest (Borjigin et al., 2013) have reported increase in cortical EEG gamma oscillations. Since prolonged hypercapnia also causes death, our findings highlight the need for further studies to determine the underlying oscillatory nature, if any, of the cortical signals associated with panic caused by $\mathrm{CO}_{2}$ inhalation.

\section{OCCLUSION OF $\mathrm{CO}_{2}$-INDUCED INCREASE IN CORTICAL ACTIVITY BY PRIOR EXPOSURE TO STRESS}

Panic attacks are more persistent in depression and stress-related psychiatric disorders (Nixon and Bryant, 2003), suggesting common or convergent pathways. The link between chronic immobilization and depression-like behavior has also been reported in rats and transgenic mouse models (Govindarajan et al., 2006; Suvrathan et al., 2010). Interestingly, we find that prior exposure to chronic stress occludes the effects of $\mathrm{CO}_{2}$ inhalation, which is manifested as a smaller change in cortical cerebral blood flow compared to unstressed animals (Figure 3B, Supplementary Figure S2). This might arise for two reasons. First, the somatosensory cortex in stressed subjects might be desensitized. The baseline activity might already be very high in stressed subjects, possibly approaching saturation, leaving little room for further enhancement by $\mathrm{CO}_{2}$ inhalation. Stress might also down regulate several signal transduction mechanisms implicated in $\mathrm{CO}_{2}$ detection (Sun et al., 2009; Wang et al., 2010). This could explain the relatively small increases induced by $\mathrm{CO}_{2}$ inhalation in stressed rats. Second, since we monitored cerebral blood flow, the lack of increase in activity might also imply a low cerebrovascular reserve capacity (Gambhir et al., 1997). However, our analysis shows that the decrease in activity results mainly from decreases in the active area rather than change per unit area (Figures 3C-E). This makes the first explanation more plausible.

The results presented here also raise the possibility that $\mathrm{CO}_{2}$ inhalation affects neural activity distributed across neural networks that have not been the traditional focus of rodent models of stress, anxiety and panic responses. The fact that we found no detectable changes within the amygdala does not rule out the possibility that projections from this structure to other brain areas, such as the somatosensory cortex, are not affected by the perturbations (stress and $\mathrm{CO}_{2}$ inhalation) used here. Indeed, growing evidence points to stress-induced modulation of connectivity between brain areas affected by repeated stress. For instance, a recent study (Ghosh et al., 2013) has shown that functional connectivity from the lateral amygdala to hippocampal area CA1 grows stronger, while directional coupling from area CA3 to CA1 within the hippocampus become weaker after chronic stress. Another study (Commins and O'Mara, 2000) found that stress facilitates the induction of long-term depression in the output regions of the hippocampal formation, thereby 
causing an effective decrease in connectivity. Future studies will be required to analyze how the spatiotemporal dynamics of interactions between cortical and subcortical areas give rise to brain activity changes seen following $\mathrm{CO}_{2}$ inhalation and consequent panic attacks.

Finally, our results using a rodent model are consistent with a recent report that, in humans, the internal threat signaled by $\mathrm{CO}_{2}$ can indeed elicit fear and panic despite the absence of an intact amygdala (Feinstein et al., 2013). This has led to the suggestion that $\mathrm{CO}_{2}$ might engage interoceptive afferent sensory pathways, which are distinct from pathways activated by exteroceptive stimuli. Furthermore, $\mathrm{CO}_{2}$ and $\mathrm{pH}$-sensitive chemoreceptors are present in many brain areas outside the amygdala including the somatosensory cortex (Wemmie, 2011). Thus, the increased activity seen in the cortex may also be due to direct activation by $\mathrm{CO}_{2}$.

\section{CONCLUSION}

A preponderance of prior evidence suggests that the panic response is mediated via an amygdalar fear pathway. However, a recent study suggests the presence of alternate and collateral pathways mediating fear and panic (Feinstein et al., 2013), independent of the amygdala under $\mathrm{CO}_{2}$ challenge. Here we imaged regions of the brain thought to play an important role in the panic response using $\mathrm{CO}_{2}$ challenge after stress induction. We found widespread changes cortical activation in unstressed animals, paradoxically not present in chronically-stressed animals, presumably because prior stress has already saturated cortical responsivity.

\section{AUTHOR CONTRIBUTIONS}

Conceived and designed the experiments: Mohammed Mostafizur Rahman, Christian M. Kerskens, Sumantra Chattarji, and Shane M. O'Mara. Performed the experiments: Mohammed Mostafizur Rahman and Christian M. Kerskens. Analyzed the data: Mohammed Mostafizur Rahman. Contributed reagents/materials/analysis tools: Shane M. O'Mara. Wrote the paper: Mohammed Mostafizur Rahman, Sumantra Chattarji, and Shane M. O’Mara.

\section{ACKNOWLEDGMENTS}

This work was supported by Science Foundation Ireland's STTF programme and the Department of Biotechnology, Government of India.

\section{SUPPLEMENTARY MATERIAL}

The Supplementary Material for this article can be found online at: http://www.frontiersin.org/journal/10.3389/fnbeh. 2014.00311/abstract

\section{REFERENCES}

Albrecht, J., Kopietz, R., Frasnelli, J., Wiesmann, M., Hummel, T., and Lundström, J. N. (2010). The neuronal correlates of intranasal trigeminal function-an ALE meta-analysis of human functional brain imaging data. Brain Res. Rev. 62, 183-196. doi: 10.1016/j.brainresrev.2009.11.001

American Psychiatric Association, American Psychiatric Association, and DSM-5 Task Force. (2013). Diagnostic and Statistical Manual of Mental Disorders: DSM5. Available online at: http://dsm.psychiatryonline.org/book. aspx?bookid $=556$. (Accessed: April 28, 2014).
Bittencourt, A. S., Nakamura-Palacios, E. M., Mauad, H., Tufik, S., and Schenberg, L. C. (2005). Organization of electrically and chemically evoked defensive behaviors within the deeper collicular layers as compared to the periaqueductal gray matter of the rat. Neuroscience 133, 873-892. doi: 10.1016/j.neuroscience.2005.03.012

Borjigin, J., Lee, U., Liu, T., Pal, D., Huff, S., Klarr, D., et al. (2013). Surge of neurophysiological coherence and connectivity in the dying brain. Proc. Natl. Acad. Sci. U.S.A. 110, 14432-14437. doi: 10.1073/pnas.1308285110

Bragin, E. O., Yeliseeva, Z. V., Vasilenko, G. F., Meizerov, E. E., Chuvin, B. T., and Durinyan, R. A. (1984). Cortical projections to the periaqueductal grey in the cat: a retrograde horseradish peroxidase study. Neurosci. Lett. 51, 271-275. doi: 10.1016/0304-3940(84)90563-9

Chung, W.-S., Farley, J. M., Swenson, A., Barnard, J. M., Hamilton, G., Chiposi, R., et al. (2010). Extracellular acidosis activates ASIC-like channels in freshly isolated cerebral artery smooth muscle cells. Am. J. Physiol. Cell Physiol. 298, C1198-C1208. doi: 10.1152/ajpcell.00511.2009

Commins, S., and O'Mara, S. M. (2000). Interactions between paired-pulse facilitation, low-frequency stimulation, and behavioral stress in the pathway from hippocampal area CA1 to the subiculum: dissociation of baseline synaptic transmission from paired-pulse facilitation and depression of the same pathway. Psychobiology 28, 1-11. doi: 10.3758/BF03330624

Coryell, W. (1997). Hypersensitivity to carbon dioxide as a disease-specific trait marker. Biol. Psychiatry 41, 259-263. doi: 10.1016/S0006-3223(97)87457-4

Critchley, H. D. (2005). Neural mechanisms of autonomic, affective, and cognitive integration. J. Comp. Neurol. 493, 154-166. doi: 10.1002/cne.20749

Dixon, W. T., Du, L. N., Faul, D. D., Gado, M., and Rossnick, S. (1986). Projection angiograms of blood labeled by adiabatic fast passage. Magn. Reson. Med. 3, 454-462. doi: 10.1002/mrm.1910030311

Feinstein, J. S., Buzza, C., Hurlemann, R., Follmer, R. L., Dahdaleh, N. S., Coryell, W. H., et al. (2013). Fear and panic in humans with bilateral amygdala damage. Nat. Neurosci. 16, 270-272. doi: 10.1038/nn.3323

Forsyth, J. P., Eifert, G. H., and Canna, M. A. (2000). Evoking analogue subtypes of panic attacks in a nonclinical population using carbon dioxide-enriched air. Behav. Res. Ther. 38, 559-572. doi: 10.1016/S0005-7967(99)00074-1

Gambhir, S., Inao, S., Tadokoro, M., Nishino, M., Ito, K., Ishigaki, T., et al. (1997). Comparison of vasodilatory effect of carbon dioxide inhalation and intravenous acetazolamide on brain vasculature using positron emission tomography. Neurol. Res. 19, 139-144.

Ghosh, S., Laxmi, T. R., and Chattarji, S. (2013). Functional connectivity from the amygdala to the hippocampus grows stronger after stress. J. Neurosci. 33, 7234-7244. doi: 10.1523/JNEUROSCI.0638-13.2013

Gorman, J. M., Kent, J. M., Sullivan, G. M., and Coplan, J. D. (2000). Neuroanatomical hypothesis of panic disorder, revised. Am. J. Psychiatry 157, 493-505. doi: 10.1176/appi.ajp.157.4.493

Gorman, J. M., Papp, L. A., Coplan, J. D., Martinez, J. M., Lennon, S., Goetz, R. R., et al. (1994). Anxiogenic effects of CO2 and hyperventilation in patients with panic disorder. Am. J. Psychiatry 151, 547-553.

Govindarajan, A., Rao, B. S. S., Nair, D., Trinh, M., Mawjee, N., Tonegawa, S., et al. (2006). Transgenic brain-derived neurotrophic factor expression causes both anxiogenic and antidepressant effects. Proc. Natl. Acad. Sci. U.S.A. 103, 13208-13213. doi: 10.1073/pnas.0605180103

Gozzi, A., Jain, A., Giovannelli, A., Giovanelli, A., Bertollini, C., Crestan, V., et al. (2010). A neural switch for active and passive fear. Neuron 67, 656-666. doi: 10.1016/j.neuron.2010.07.008

Griez, E., and Schruers, K. (1998). Experimental pathophysiology of panic. J. Psychosom. Res. 45, 493-503. doi: 10.1016/S0022-3999(98)00027-0

Griffin, K. M., Blau, C. W., Kelly, M. E., O’Herlihy, C., O’Connell, P. R., Jones, J. F. X., et al. (2010). Propofol allows precise quantitative arterial spin labelling functional magnetic resonance imaging in the rat. Neuroimage 51, 1395-1404. doi: 10.1016/j.neuroimage.2010.03.024

Groome, D., and Soureti, A. (2004). Post-traumatic stress disorder and anxiety symptoms in children exposed to the 1999 Greek earthquake. Br. J. Psychol. 95, 387-397. doi: 10.1348/0007126041528149

Hennig, J., Nauerth, A., and Friedburg, H. (1986). RARE imaging: a fast imaging method for clinical MR. Magn. Reson. Med. 3, 823-833. doi: 10.1002/mrm.1910030602

Jenck, F., Moreau, J.-L., and Martin, J. R. (1995). Dorsal periaqueductal grayinduced aversion as a simulation of panic anxiety: elements of face and predictive validity. Psychiatry Res. 57, 181-191. doi: 10.1016/0165-1781(95)02673-K 
Kelly, M. E., Blau, C. W., and Kerskens, C. M. (2009). Bolus-tracking arterial spin labelling: theoretical and experimental results. Phys. Med. Biol. 54, 1235-1251. doi: 10.1088/0031-9155/54/5/009

Kerskens, C. M., Hoehn-Berlage, M., Schmitz, B., Busch, E., Bock, C., Gyngell, M. L., et al. (1996). Ultrafast perfusion-weighted MRI of functional brain activation in rats during forepaw stimulation: comparison with T2 -weighted MRI. NMR Biomed. 9, 20-23.

Killackey, H. P., and Sherman, S. M. (2003). Corticothalamic projections from the rat primary somatosensory cortex. J. Neurosci. 23, 7381-7384.

Kiser, R. S., Brown, C. A., Sanghera, M. K., and German, D. C. (1980). Dorsal raphe nucleus stimulation reduces centrally-elicited fearlike behavior. Brain Res. 191, 265-272. doi: 10.1016/0006-8993(80)90331-5

Klein, D. F. (1993). False suffocation alarms, spontaneous panics, and related conditions. An integrative hypothesis. Arch. Gen. Psychiatry 50, 306-317. doi: 10.1001/archpsyc.1993.01820160076009

Kumar, K., Toth, C., and Nath, R. K. (1997). Deep brain stimulation for intractable pain: a 15-year experience. Neurosurgery 40, 736-746. discussion: 746-747. doi: 10.1097/00006123-199704000-00015

Lakshminarasimhan, H., and Chattarji, S. (2012). Stress leads to contrasting effects on the levels of brain derived neurotrophic factor in the hippocampus and amygdala. PLOS ONE 7:e30481. doi: 10.1371/journal.pone. 0030481

Mitra, R., Jadhav, S., McEwen, B. S., Vyas, A., and Chattarji, S. (2005). Stress duration modulates the spatiotemporal patterns of spine formation in the basolateral amygdala. Proc. Natl. Acad. Sci. U.S.A. 102, 9371-9376. doi: 10.1073/pnas.0504011102

Morgan III, C. A., Grillon, C., Southwick, S. M., Davis, M., and Charney, D. S. (1995). Fear-potentiated startle in posttraumatic stress disorder. Biol. Psychiatry 38, 378-385. doi: 10.1016/0006-3223(94)00321-S

Nixon, R. D. V., and Bryant, R. A. (2003). Peritraumatic and persistent panic attacks in acute stress disorder. Behav. Res. Ther. 41, 1237-1242. doi: 10.1016/S00057967(03)00150-5

O’Hearn, E., and Molliver, M. E. (1984). Organization of raphe-cortical projections in rat: a quantitative retrograde study. Brain Res. Bull. 13, 709-726.

Papp, L. A., Martinez, J. M., Klein, D. F., Coplan, J. D., Norman, R. G., Cole, R., et al. (1997). Respiratory psychophysiology of panic disorder: three respiratory challenges in 98 subjects. Am. J. Psychiatry 154, 1557-1565.

Paxinos, G., and Watson, C. (2005). The Rat Brain in Stereotaxic Coordinates. Burlington, MA: Elsevier Academic Press.

Preter, M., and Klein, D. F. (2008). Panic, suffocation false alarms, separation anxiety and endogenous opioids. Prog. Neuropsychopharmacol. Biol. Psychiatry 32, 603-612. doi: 10.1016/j.pnpbp.2007.07.029

Sakai, Y., Kumano, H., Nishikawa, M., Sakano, Y., Kaiya, H., Imabayashi, E., et al. (2005). Cerebral glucose metabolism associated with a fear network in panic disorder. Neuroreport 16, 927-931. doi: 10.1097/00001756-20050621000010

Schenberg, L. C., Bittencourt, A. S., Sudré, E. C. M., and Vargas, L. C. (2001). Modeling panic attacks. Neurosci. Biobehav. Rev. 25, 647-659. doi: 10.1016/S0149-7634(01)00060-4

Schenberg, L. C., Póvoa, R. M. F., Costa, A. L. P., Caldellas, A. V., Tufik, S., and Bittencourt, A. S. (2005). Functional specializations within the tectum defense systems of the rat. Neurosci. Biobehav. Rev. 29, 1279-1298. doi: 10.1016/j.neubiorev.2005.05.006

Schimitel, F. G., de Almeida, G. M., Pitol, D. N., Armini, R. S., Tufik, S., and Schenberg, L. C. (2012). Evidence of a suffocation alarm system within the periaqueductal gray matter of the rat. Neuroscience 200, 59-73. doi: 10.1016/j.neuroscience.2011.10.032

Schneider, C. A., Rasband, W. S., and Eliceiri, K. W. (2012). NIH Image to ImageJ: 25 years of image analysis. Nat. Methods 9, 671-675. doi: 10.1038/ nmeth.2089
Sessle, B. J., Ball, G. J., and Lucier, G. E. (1981). Suppressive influences from periaqueductal gray and nucleus raphe magnus on respiration and related reflex activities and on solitary tract neurons, and effect of naloxone. Brain Res. 216, 145-161. doi: 10.1016/0006-8993(81)91283-X

Stein, M. B., and Asmundson, G. J. C. (1994). Autonomic function in panic disorder: cardiorespiratory and plasma catecholamine responsivity to multiple challenges of the autonomic nervous system. Biol. Psychiatry 36, 548-558. doi: 10.1016/0006-3223(94)90619-X

Sun, L., Wang, H., Hu, J., Han, J., Matsunami, H., and Luo, M. (2009). Guanylyl cyclase-D in the olfactory $\mathrm{CO} 2$ neurons is activated by bicarbonate. Proc. Natl. Acad. Sci. U.S.A. 106, 2041-2046. doi: 10.1073/pnas.0812220106

Suvrathan, A., Bennur, S., Ghosh, S., Tomar, A., Anilkumar, S., and Chattarji, S. (2014). Stress enhances fear by forming new synapses with greater capacity for long-term potentiation in the amygdala. Philos. Trans. R. Soc. B Biol. Sci. 369, 151-159. doi: 10.1098/rstb.2013.0151

Suvrathan, A., Tomar, A., and Chattarji, S. (2010). Effects of chronic and acute stress on rat behaviour in the forced-swim test. Stress 13, 533-540. doi: $10.3109 / 10253890.2010 .489978$

Vyas, A., Mitra, R., Shankaranarayana Rao, B. S., and Chattarji, S. (2002). Chronic stress induces contrasting patterns of dendritic remodeling in hippocampal and amygdaloid neurons. J. Neurosci. 22, 6810-6818.

Wang, C., Bomberg, E., Billington, C., Levine, A., and Kotz, C. M. (2007). Brain-derived neurotrophic factor in the hypothalamic paraventricular nucleus increases energy expenditure by elevating metabolic rate. Am. J. Physiol. Regul. Integr. Comp. Physiol. 293, R992-R1002. doi: 10.1152/ajpregu.005 16.2006

Wang, Y. Y., Chang, R. B., and Liman, E. R. (2010). TRPA1 Is a component of the nociceptive response to CO2. J. Neurosci. 30, 12958-12963. doi: 10.1523/JNEUROSCI.2715-10.2010

Wemmie, J. A. (2011). Neurobiology of panic and $\mathrm{pH}$ chemosensation in the brain. Dialogues Clin. Neurosci. 13, 475-483.

Wemmie, J. A., Askwith, C. C., Lamani, E., Cassell, M. D., Freeman, J. H., and Welsh, M. J. (2003). Acid-sensing ion channel 1 is localized in brain regions with high synaptic density and contributes to fear conditioning. J. Neurosci. 23, 5496-5502.

Williams, G., Bing, C., Cai, X. J., Harrold, J. A., King, P. J., and Liu, X. H. (2001). The hypothalamus and the control of energy homeostasis. Physiol. Behav. 74, 683-701. doi: 10.1016/S0031-9384(01)00612-6

Ziemann, A. E., Allen, J. E., Dahdaleh, N. S., Drebot, I. I., Coryell, M. W., Wunsch, A. M., et al. (2009). The amygdala is a chemosensor that detects carbon dioxide and acidosis to elicit fear behavior. Cell 139, 1012-1021. doi: 10.1016/j.cell.2009.10.029

Conflict of Interest Statement: The authors declare that the research was conducted in the absence of any commercial or financial relationships that could be construed as a potential conflict of interest.

Received: 09 May 2014; accepted: 25 August 2014; published online: 16 September 2014.

Citation: Rahman MM, Kerskens CM, Chattarji S and O'Mara SM (2014) Chronic immobilization stress occludes in vivo cortical activation in an animal model of panic induced by carbon dioxide inhalation. Front. Behav. Neurosci. 8:311. doi: 10.3389/ fnbeh.2014.00311

This article was submitted to the journal Frontiers in Behavioral Neuroscience.

Copyright (c) 2014 Rahman, Kerskens, Chattarji and O'Mara. This is an openaccess article distributed under the terms of the Creative Commons Attribution License (CC BY). The use, distribution or reproduction in other forums is permitted, provided the original author(s) or licensor are credited and that the original publication in this journal is cited, in accordance with accepted academic practice. No use, distribution or reproduction is permitted which does not comply with these terms. 